EPJ Web of Conferences 31, 00023 (2012)

DOI: 10.1051/epjconf/20123100023

(C) Owned by the authors, published by EDP Sciences - SIF, 2012

\title{
Interplay between collision dynamics and nuclear matter properties from microscopic viewpoint
}

\author{
AKIRA ONO \\ Department of Physics, Tohoku University, Sendai 980-8578, Japan
}

\begin{abstract}
Nuclear matter properties have been explored through heavy-ion collisions. Studies with microscopic dynamical models, such as antisymmetrized molecular dynamics, are reviewed putting some emphasis on fragmentation and clusterization.
\end{abstract}

\section{Introduction}

Through the study of heavy-ion collisions, nuclear matter properties have been explored, such as the equation of state and liquid-gas phase transition. Many-body correlations are also an important aspect of matter properties. In particular, correlations to form clusters and fragments appear in low density systems as a consequence of the nuclear saturation property, which has to be understood quantum-mechanically. An important question is how the matter properties influence on the collision dynamics and on the final results. Inversely, it is also important to know what kind of nuclear matter is produced during collisions which often look quite dynamical. For example, it is not a trivial question whether any kind of equilibrium is relevant in collisions. Microscopic models have been useful to answer these questions. 


\section{Microscopic approaches}

Antisymmetrized molecular dynamics (AMD) [1,2] employs Gaussian wave packets with a fixed width as single-particle wave functions. The time evolution of the wave packet centroids are determined by an equation of motion that includes some stochastic terms. The wave packets move in the mean field calculated from the adopted effective interaction and collide with each other by the residual interaction. Some in-medium cross section is assumed and the Pauli-blocking is taken into account. Furthermore, each wave packet will be split by random displacements to the centroid in the phase space based on the change of the shape and width of the phase space distribution. An important model ingredient is the coherence time $\tau$ during which the coherent single-particle motion under the mean field is considered until the decoherence. When the wave packet splitting was first introduced, we took the limit of $\tau \rightarrow 0$ which corresponds to the strongest decoherence. More recently, the formulation was generalized to allow finite coherence time [3]. A reasonable choice may be to consider the decoherence for each nucleon when it collides with another nucleon $\left(\tau=\tau_{\mathrm{NN}}\right)$.

The AMD approach with stochastic extensions is conceptually very similar to some mean-field approaches with the inclusion of fluctuations on the one-body distribution function $f$. The stochastic mean field (SMF) model by Colonna et al. assumes that the effect of the fluctuation term in the Boltzmann-Langevin equation is to induce fluctuations on $f$ in such a way that the variance of $f$ in each phase-space cell is given by $\Delta f^{2}=\bar{f}(1-\bar{f})[4]$. This means that $f$ takes values of 1 (fully occupied) or 0 (empty) in each phase-space cell. If each occupied cell $(f=1)$ is identified with a wave packet, SMF is conceptually very similar to AMD. However, different approximations are introduced in different approaches, so the results should be carefully examined.

\section{Systems in thermal equilibrium}

By solving the time evolution of a many-body system in a container for a very long time, a microcanonical ensemble can be generated for the given energy and volume. In Refs. [5,6], Furuta and Ono performed AMD calculations to obtain constant-pressure caloric curves in which phase transition should be clearly identified. Wave packet splitting was considered with a densitydependent coherence time $\tau(\rho)$ in this study. For a calculated microcanonical ensemble of a given energy $E$ and a volume $V$, the temperature $T$ and the 


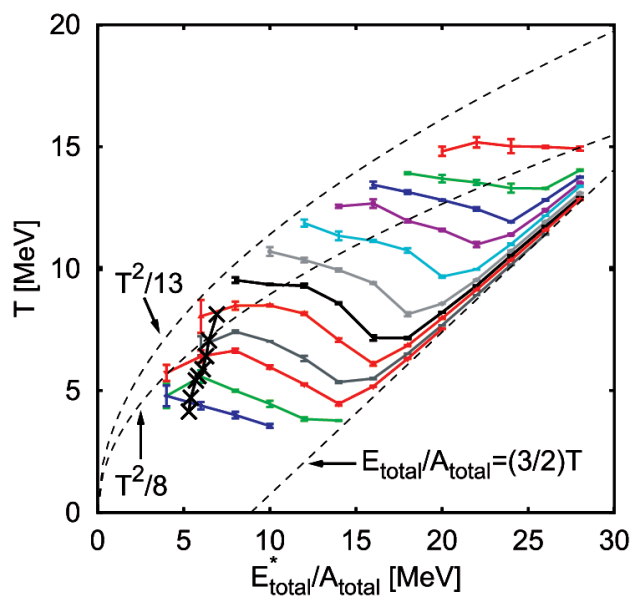

Figure 1: Constant pressure caloric curves calculated with AMD for the $A=36$ system. The cross symbols indicate the equilibrium state corresponding to the reaction system from $t=80$ to $300 \mathrm{fm} / c$ for ${ }^{40} \mathrm{Ca}+{ }^{40} \mathrm{Ca}$ central collisions. From Ref. [6].

pressure $P$ are defined by using the kinetic energies of gas-like nucleons and the information of the reflections of particles at the boundary of the container, respectively. Then caloric curves are drawn as $T(E)$ for different values of $P$. The result for the system of $N=18$ and $Z=18$ is shown in Fig. 1. Nuclear liquid-gas phase transition, as a first order phase transition, is clearly seen in this result as the back-bending of caloric curves.

The construction of microcanonical ensembles can also be regarded as a way to explore the many-body states at various excitation energies, as illustrated in Fig. 2. The density profile at each of the excitation energies $E^{*} / A=4,10$ and $28 \mathrm{MeV}$ is a sample taken from the calculated microcanonical ensemble [5]. At low excitation energies, a single large nucleus is usually observed. The energy $E^{*} / A=10 \mathrm{MeV}$ is in the region of liquidgas phase transition where the density of states $W(E)$ shows an anomalous behavior and each density profile typically shows several nuclei. When the energy is further raised to $28 \mathrm{MeV} /$ nucleon, for example, the state may be regarded as a gas state but the gas is composed of clusters as well as nucleons. Experimental data have shown that the pure nucleon gas is never realized at this energy region [7].

Thus AMD can describe both dynamical reactions in heavy-ion collisions and virtually equilibrated systems reasonably well. In Ref. [6], we have investigated the question whether equilibrium is really relevant in multifrag- 


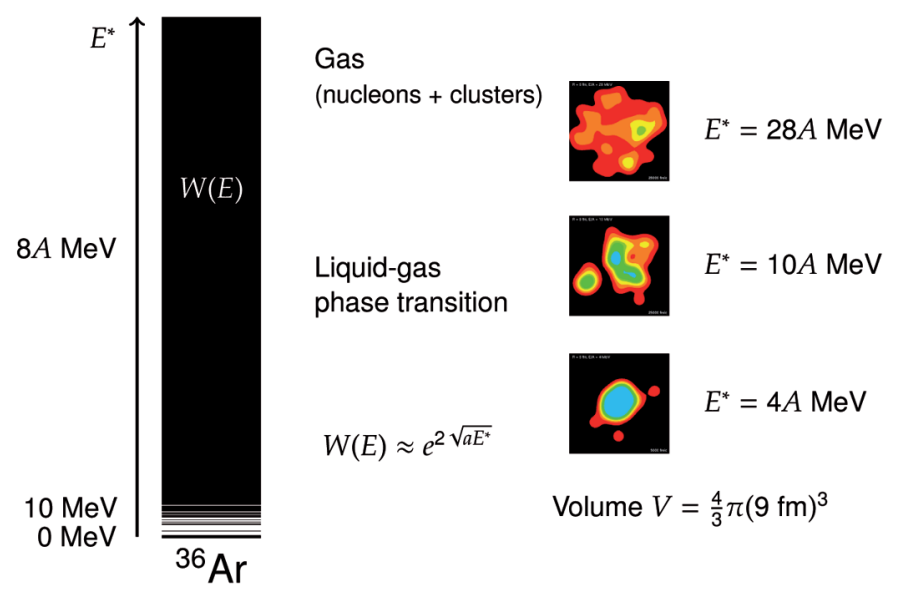

Figure 2: Excited states of a many-nucleon system with $N=Z=18$ confined in a virtual spherical container with a radius of $9 \mathrm{fm}$. The density distribution at each excitation energy $\left(E^{*} / A=4,10\right.$ and $\left.28 \mathrm{MeV}\right)$ shows a snapshot taken from the AMD equilibrium calculation of Ref. [5].

mentation by comparing the details of reaction calculations and equilibrium calculations performed by the same AMD model. The calculations show that there exists an equilibrium ensemble which well reproduces the reaction ensemble at each reaction time $t$ for the investigated period $80 \leq t \leq 300$ $\mathrm{fm} / \mathrm{c}$ in ${ }^{40} \mathrm{Ca}+{ }^{40} \mathrm{Ca}$ central collisions at $35 \mathrm{MeV} /$ nucleon, as far as fragment observables (fragment yields and excitation energies) are concerned. Thus the corresponding temperature and excitation energy (or the volume and pressure) can be identified at each reaction time. In Fig. 1, the path of the reaction form $t=80$ to $300 \mathrm{fm} / c$ is drawn by the cross symbols. It is also important to note that there are some other observables which show discrepancies between the reaction and equilibrium ensembles [6].

\section{Collective dynamics and multifragmentation}

\subsection{Radial oscillations}

Radial breathing oscillations of nuclei are interesting particularly because they are closely related to the compressibility of nuclei and nuclear matter. AMD can be a powerful tool to explore the radial oscillations at various amplitudes. The large amplitude oscillations should be continuously linked to the expansion of nuclei without restoration at higher energies where many- 


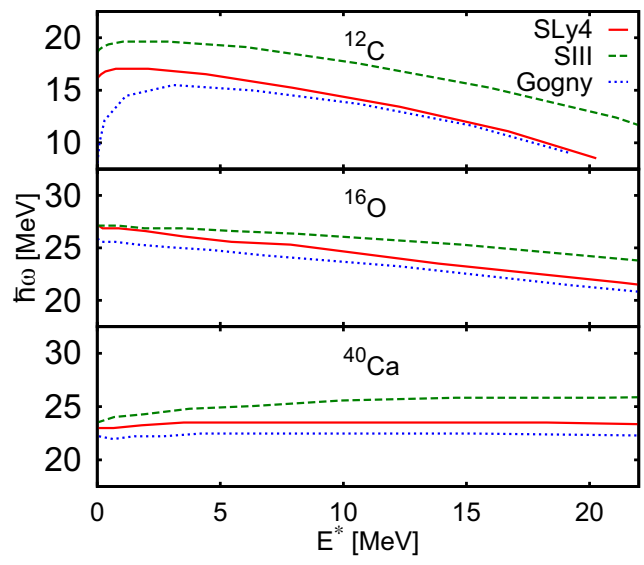

Figure 3: AMD prediction for the frequency $\hbar \omega$ of the radial oscillation as a function of the oscillation energy for ${ }^{12} \mathrm{C},{ }^{16} \mathrm{O}$ and ${ }^{40} \mathrm{Ca}$ with the SLy4, SIII and Gogny interactions. The nuclear matter incompressibilities for these interactions are $K=$ 230, 355 and $228 \mathrm{MeV}$, respectively. From Ref. [8].

body correlations play important roles in forming clusters and fragment nuclei.

The radial oscillations of the ${ }^{12} \mathrm{C}$ and other nuclei were calculated with AMD without any stochastic terms in Ref. [8]. Figure 3 shows the dependence of the oscillation frequency on the amplitude or the excitation energy. The results for three different effective interactions are shown. Dependence on the incompressibility is clearly observed. The dependence on the amplitude suggests the unharmomicity of the oscillation.

The same problem was studied by the fermionic molecular dynamics (FMD) as well [8], in which the width parameters of individual wave packets are treated as time-dependent variables as well as the centroid variables. In this case, the calculated result shows an oscillation pattern composed of two modes with different frequencies. It has turned out that one of the two modes corresponds to the change of the wave packet widths and the other corresponds to the motion of the wave packet centroids. The latter mode is the same as that observed in the AMD result and related to the $\alpha$-clustering degrees of freedom. The former mode is the breathing of individual singleparticle wave packets. Thus the single-particle excitation and the clustering excitation are both relevant in radially oscillating systems. 


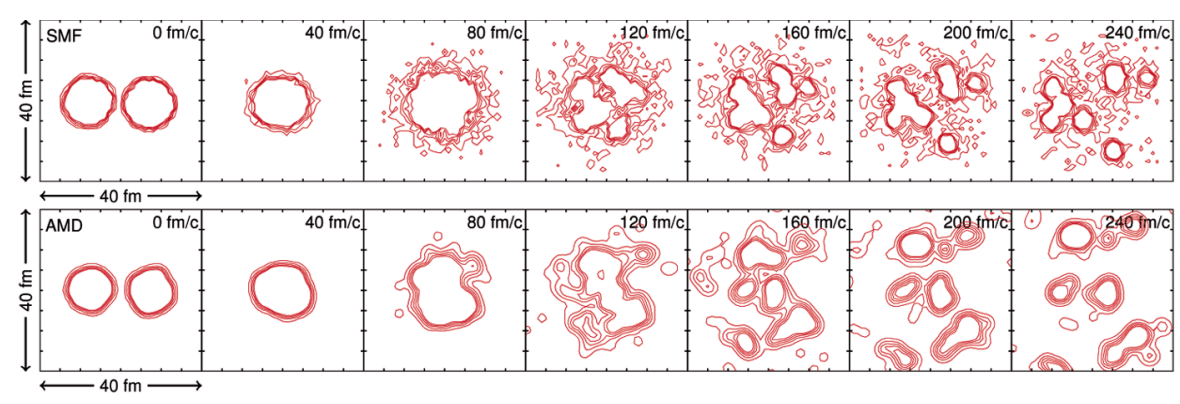

Figure 4: Time evolution of density profiles obtained by SMF (upper) and AMD (lower), for central ${ }^{112} \mathrm{Sn}+{ }^{112} \mathrm{Sn}$ collisions at $50 \mathrm{MeV} /$ nucleon. From Ref. [10].

\subsection{Expansion followed by fragmentation}

Figure 4 shows a typical example of the time evolution of ${ }^{112} \mathrm{Sn}+{ }^{112} \mathrm{Sn}$ central collisions at $50 \mathrm{MeV} /$ nucleon $[9,10]$. The calculation by the SMF model in the upper row is compared with the AMD calculation in the lower row. In both cases, the system compressed at an early stage starts to expand almost spherically. As the expansion proceeds, the density fluctuation develops to form many fragments.

As discussed in a previous section, these two models are conceptually similar, but the results can be different due to the different approximate treatments of fluctuations. In fact, it is observed that the density fluctuation (among different events) is already developing in AMD at the relatively early stage of $50 \lesssim t \lesssim 100 \mathrm{fm} / c$, while the fluctuation develops in SMF only at a later stage $t \sim 100 \mathrm{fm} / c$ suggesting a fragmentation mechanism by spinodal decomposition. Thus the many-body correlations are stronger in AMD. This difference can be interpreted as the origin of the differences in the expansion velocity, the nucleon emission and so on predicted by these models $[9,10]$.

The fragmentation of expanding systems has been observed in experiments in a wide range of incident energies of central heavy-ion collisions $[11,12]$. Strong expansion is suggested by the observed almost linear dependence of the fragment kinetic energy as a function of the fragment size.

\subsection{Stopping}

The shape of the expanding system is most importantly characterized by the degree of stopping, which may be quantified by $R_{E}=\frac{1}{2} \sum\left(E_{x}+E_{y}\right) / \sum E_{z}$ where the summations are for the kinetic energy components of all the charged particles. A recent experimental data [13] shows that the stop- 
ping is moderate $\left(R_{E} \approx 0.5-0.6\right)$ in the energy region from 30 to 100 $\mathrm{MeV} /$ nucleon. As naturally expected, stopping depends on the in-medium two-nucleon collision cross sections in transport model calculations. Some AMD calculations have also shown that the stiffness of the equation of state influences stopping as well [14].

As stopping is an important aspect to determine the global dynamics, the model ingredients should be constrained to reproduce it before comparing other observables with experimental data. By changing the two-nucleon cross sections, however, an AMD calculation shows that the fragment yields depend on stopping only very weakly in a specific reaction of $\mathrm{Sn}+\mathrm{Sn}$ central collisions at $50 \mathrm{MeV} /$ nucleon.

\subsection{Yields of fragments and clusters}

Figure 5 shows the AMD results of the fragment change distribution compared with data [3]. The result depends very much on the choice of the coherence time. In this reaction system, the fragment yields for $Z \gtrsim 3$ are well reproduced by AMD when the wave packet splitting is introduced with the coherence time $\tau=\tau_{\mathrm{NN}}$. It is often convenient to define the liquid and gas parts of the system as the parts composed of $Z \geq 3$ fragments and $Z \leq 2$ particles, respectively. The comparison shows that the total charge of the liquid part, $Z_{\text {liq }}$, in the AMD result (with $\tau=\tau_{\mathrm{NN}}$ ) is consistent with the experimental data, and therefore the total charge of the gas part, $Z_{\text {gas }}=Z_{\text {system }}-Z_{\text {liq }}$, is also consistent.

However, a problem is found in the composition of the gas part in the result with $\tau=\tau_{\mathrm{NN}}$. The $\alpha$-particle multiplicity $M_{\alpha} \approx 7$ is too small and the proton multiplicity $M_{p} \approx 20$ is too large compared with the experimental data $M_{\alpha} \approx M_{p} \approx 10$ [11]. It should be noted that only about $10 \%$ of the total protons in the system is emitted as free protons in this reaction at $50 \mathrm{MeV} /$ nucleon. It is also known experimentally that still a half of the protons are bound in clusters even at $1 \mathrm{GeV} /$ nucleon. Thus the experimental data have been suggesting the importance of cluster correlations. The comparison with the data indicates that the AMD approach with the wave packet splitting, which is largely based on the single-particle motion in the mean field, does not include sufficient cluster correlations in the dynamics. 


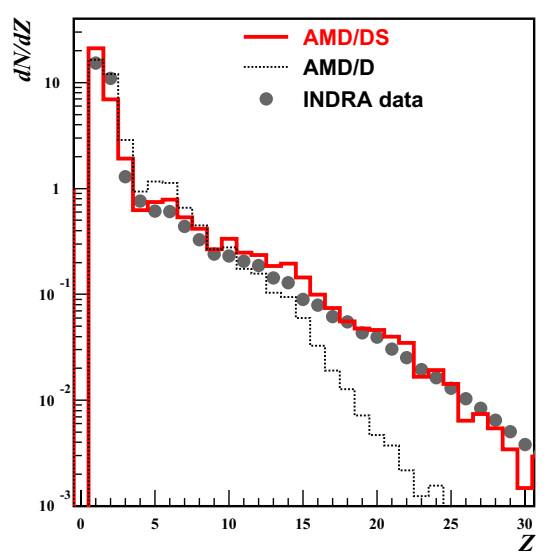

Figure 5: The fragment charge distribution in ${ }^{129} \mathrm{Xe}+\mathrm{Sn}$ collisions at 50 $\mathrm{MeV} /$ nucleon with the impact parameter $0<b<4 \mathrm{fm}$, after calculating the secondary decay of excited clusters and applying the experimental filter for the detector setup. Solid histogram (labeled AMD/DS) shows the result of AMD with the coherence time $\tau=\tau_{\mathrm{NN}}$, while the dotted histogram (labeled AMD/D) shows the result with the strongest decoherence $\tau \rightarrow 0$. The INDRA experimental data are shown by solid points. From Ref. [3].

\section{Cluster correlations in multifragmentation}

\subsection{Incorporation of cluster correlations in AMD}

In order to go beyond a standard picture of mean field plus two-nucleon collisions, we explicitly introduce cluster correlations into the time evolution of AMD by considering the cluster formation in the final state of each twonucleon collision. We consider a process $\mathrm{N}_{1}+\mathrm{N}_{2}+\mathrm{B}_{1}+\mathrm{B}_{2} \rightarrow \mathrm{C}_{1}+\mathrm{C}_{2}$, where two nucleons $\mathrm{N}_{1}$ and $\mathrm{N}_{2}$ interacts by the residual interaction and each of them forms a cluster with spectator nucleon(s) $\mathrm{B}_{i}(i=1,2)$. Formed clusters are denoted by $\mathrm{C}_{i}\left(=\mathrm{N}_{i}+\mathrm{B}_{i}\right)$. A kind of impulse approximation is employed to estimate the cluster formation probabilities in a similar way to Danielewicz et al. [15]. Namely, the cluster formation cross section is given by the product of a kinematical factor, the usual two-nucleon collision cross section and the overlap probabilities that each collided nucleon $\mathrm{N}_{i}$ forms a cluster with spectator nucleon(s) $\mathrm{B}_{i}$. We consider the formation of clusters composed of $1,2,3$, and 4 nucleons.

The time evolution of nucleons in formed clusters are calculated almost as usual in AMD, except that the fluctuation of wave packet splitting is turned off for the internal degrees of freedom of each cluster. This is because the clusters are assumed to be stable under the mean field effect (except for 


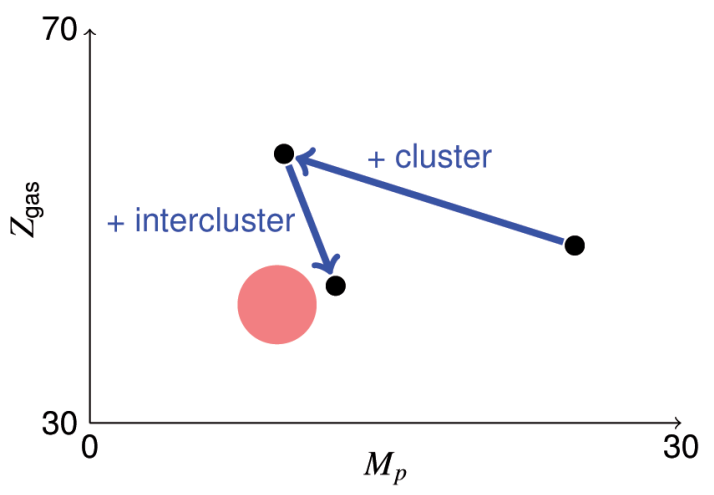

Figure 6: The effects of cluster correlations and intercluster correlations on the proton multiplicity $M_{\alpha}$ and the number of protons in the gas $Z_{\text {gas }}$. The filled big circle shows the region consistent with experimental data.

the dependence of the force on the spin and the isospin). A cluster can be broken by a collision with another nucleon or cluster.

The cluster correlations have drastic effects on the final yields. In a calculation of the ${ }^{112} \mathrm{Sn}+{ }^{112} \mathrm{Sn}$ central collisions, $M_{p}$ decreases from 25 to 10 and $M_{\alpha}$ increases from 6 to 15 by the inclusion of cluster correlations. Furthermore, $Z_{\text {gas }}$ increases from 48 to 57 and $Z_{\text {liq }}$ decreases accordingly. In short, the tendency of cluster gas becomes strong because of the change of the energy balance and the number of the effective degrees of freedom. This tendency is too strong compared with experimental data where $Z_{\text {gas }}$ is between 40 and 50 .

\subsection{Intercluster correlations}

The cluster correlations have to be incorporated in addition to the usual AMD dynamics. This is because the nucleons in a cluster are loosely bound so that there is only one bound state. For the same reason, the correlations of two or several clusters require a special consideration since clusters are loosely bound in light nuclei such as $\mathrm{Li}$ and Be isotopes.

Effects of intercluster correlations are tested by introducing a stochastic process of bonding clusters. The relative momentum between clusters is replaced by zero if moderately separated clusters are moving away from each other with a small relative momentum. Thus this cluster bonding process works to select bound states of two or several clusters during the time evolution of reactions.

This intercluster correlations certainly reduce the tendency of cluster gas. The $\alpha$-particle multiplicity, $M_{\alpha}$, is reduced from 15 to 10 by the cluster 
bonding effect, while $M_{p}$ increases moderately from 10 to 12.5 . The number of the protons in the gas, $Z_{\text {gas }}$, is reduced from 57 to 44 , which is now consistent with data. The effects of cluster correlations and of intercluster correlations are depicted in Fig. 6 in the plane of $M_{p}$ and $Z_{\text {gas }}$. Though the strengths of the effects may be adjusted by introducing model parameters, the experimental data suggests the importance of both effects.

\section{Acknowledgments}

Some of the works presented here were supported by KAKENHI 21540253 from Japan Society for the Promotion of Science, and by High Energy Accelerator Research Organization (KEK) as a supercomputer project.

\section{References}

[1] A. Ono et al., Prog. Theor. Phys. 87 (1992) 1185.

[2] A. Ono and H. Horiuchi, Prog. Part. Nucl. Phys. 53 (2004) 501.

[3] A. Ono, S. Hudan, A. Chbihi and J.D. Frankland, Phys. Rev. C 66 (2002) 014603.

[4] M. Colonna et al., Nucl. Phys. A642 (1998) 449.

[5] T. Furuta and A. Ono, Phys. Rev. C 74 (2006) 014612.

[6] T. Furuta and A. Ono, Phys. Rev. C 79 (2009) 014608.

[7] B. Borderie et al., Eur. Phys. J. A 6 (1999) 197.

[8] T. Furuta et al., Phys. Rev. C 82 (2010) 034307.

[9] J. Rizzo, M. Colonna and A. Ono Phys. Rev. C 76 (2007) 024611.

[10] M. Colonna, A. Ono and J. Rizzo Phys. Rev. C 82 (2010) 054613.

[11] S. Hudan et al., Phys. Rev. C 67 (2003) 064613.

[12] W. Reisdorf et al., Nucl. Phys. A612 (1997) 493.

[13] G. Lehaut et al., Phys. Rev. Lett. 104 (2010) 232701.

[14] R. Wada et al., Phys. Rev. C 69 (2004) 044610.

[15] P. Danielewicz and G.F. Bertsch, Nucl. Phys. A533 (1991) 712. 